\title{
L'amour courtois et la symbolique de la Kénose dans le motet 10 de Guillaume de Machaut
}

Charlène Mercier, Antoine Roux, Vanessa Vermeulen et Jennifer Verpiot

\section{(2) OpenEdition \\ 1 Journals}

Édition électronique

URL : https://journals.openedition.org/cem/8442

DOI : $10.4000 /$ cem.8442

ISSN : 1954-3093

\section{Éditeur}

Centre d'études médiévales Saint-Germain d'Auxerre

\section{Édition imprimée}

Date de publication : 15 août 2008

ISSN : 1623-5770

\section{Référence électronique}

Charlène Mercier, Antoine Roux, Vanessa Vermeulen et Jennifer Verpiot, «L'amour courtois et la symbolique de la Kénose dans le motet 10 de Guillaume de Machaut », Bulletin du centre d'études médiévales d'Auxerre / BUCEMA [En ligne], 12 | 2008, mis en ligne le 09 avril 2009, consulté le 22 septembre 2022. URL : http://journals.openedition.org/cem/8442 ; DOI : https://doi.org/10.4000/cem. 8442

Ce document a été généré automatiquement le 22 septembre 2022.

\section{(c) (i) (9)}

Creative Commons - Attribution - Pas d'Utilisation Commerciale - Partage dans les Mêmes Conditions 4.0 International - CC BY-NC-SA 4.0

https://creativecommons.org/licenses/by-nc-sa/4.0/ 


\title{
L'amour courtois et la symbolique de la Kénose dans le motet 10 de Guillaume de Machaut*
}

\author{
Charlène Mercier, Antoine Roux, Vanessa Vermeulen et Jennifer Verpiot
}

Dans l'esprit médiéval, chaque chose est ordonnée par l'harmonie de Dieu, décidée selon un plan unique. Il en va ainsi du macrocosme, de l'ordre régissant l'univers tout entier, au microcosme, allant jusqu'à gouverner la structure interne de chaque création de l'intellect humain. D'après la première épître de saint Paul aux Corinthiens $(13,12)$ : «Videmus nunc per speculum in aenigmate, tunc autem facie ad faciem ${ }^{1}$. " Étudier une pièce de l'époque reviendrait-il à décrypter les liens cachés entre l'architecture spécifique de l'œuvre et celle de l'univers? Les motets du XIV ${ }^{e}$ siècle répondent particulièrement bien à cette conception : on y trouve une étroite correspondance entre texte et musique et une surprenante exploitation du symbolisme numérique. Inspirés du travail de Margaret Bent sur les motets 9 et 15 de Guillaume de Machaut ${ }^{2}$, nous avons voulu vérifier ces mêmes principes dans le motet 10 , jamais étudié de manière approfondie jusqu'à présent ${ }^{3}$. 
I. Helas! ou sera pris confors

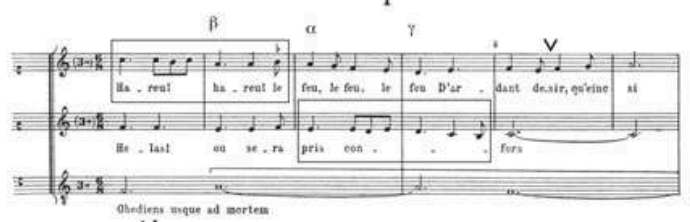
AI

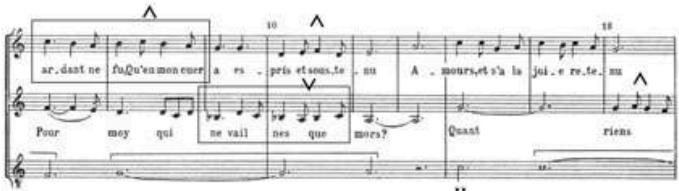

$\mathrm{R} \wedge \wedge \wedge$
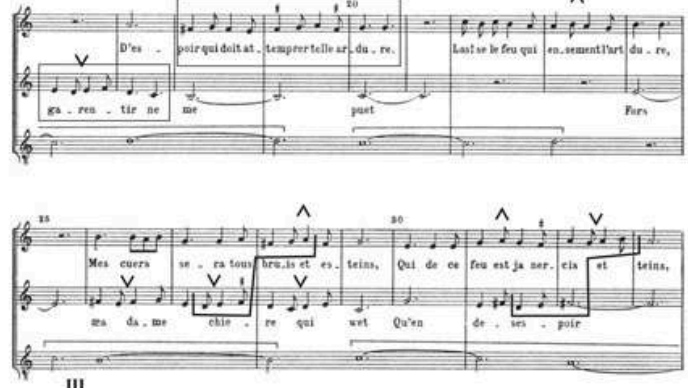

III

\begin{tabular}{|c|c|}
\hline Echanges rythmiques & Echanges mélodiques \\
\hline & \\
\hline Cellules en « V » ou « V inversé » & Cellules en miroir \\
\hline$A$ ou $\mathrm{V}$ & $\cdots \cdots$ \\
\hline Hoquet: «h.» & \\
\hline
\end{tabular}



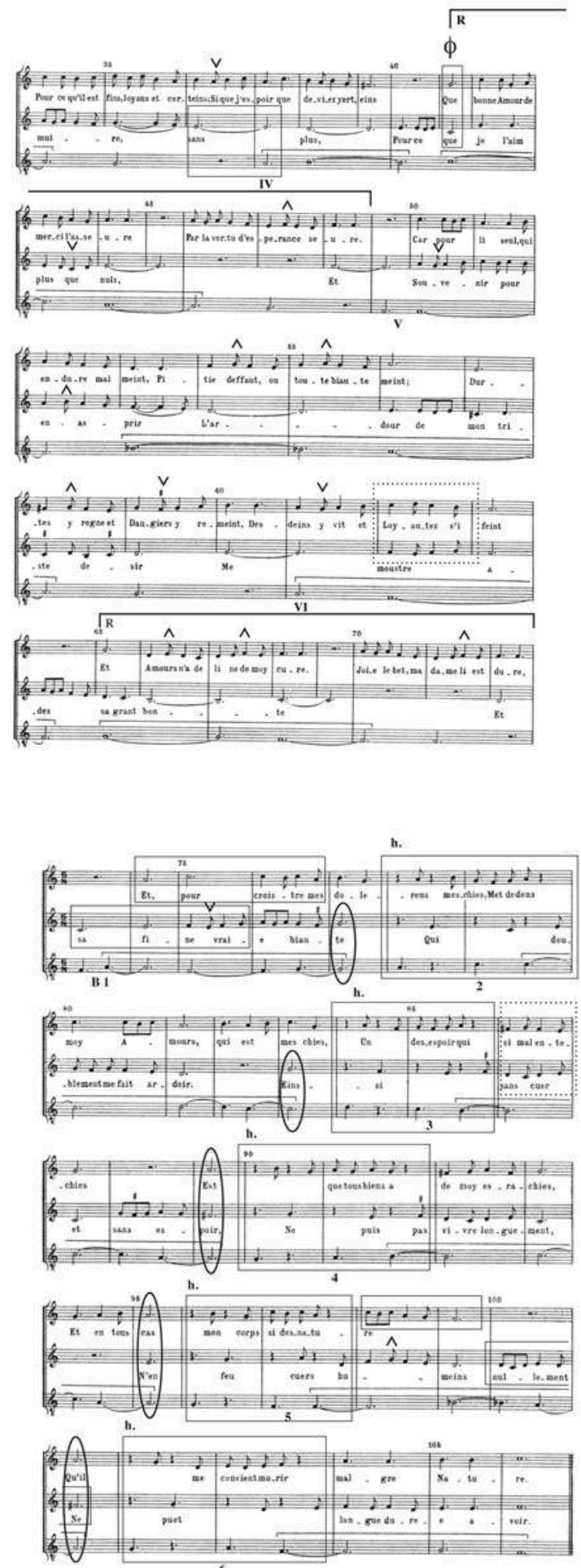


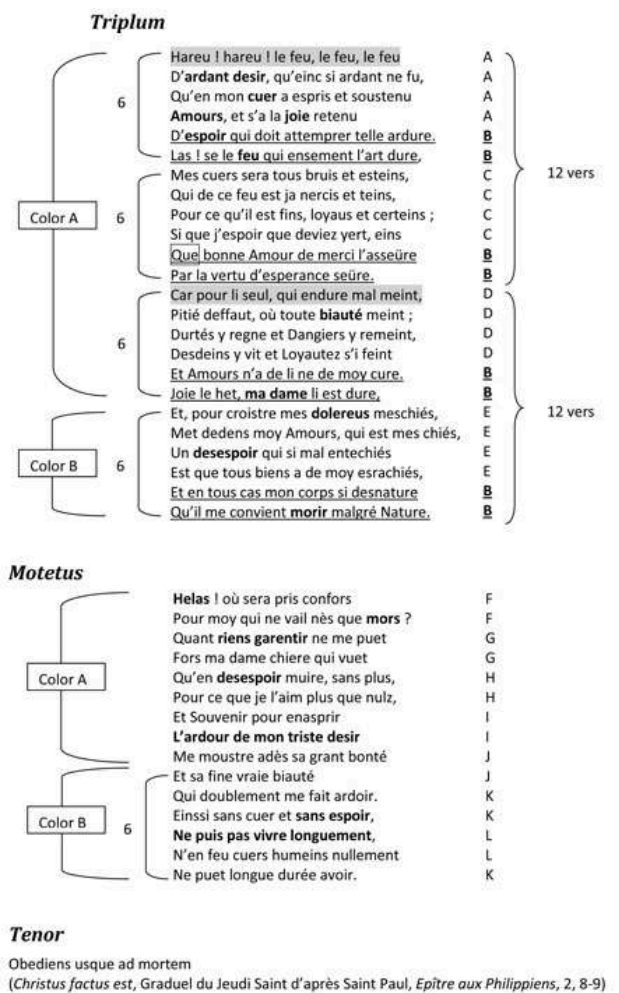

Cette pièce est un motet à trois voix, comportant des textes profanes en français aux deux voix supérieures, et un tenor liturgique en latin. Le triplum évoque l'espoir d'un amour impossible tandis que le motetus énonce le désespoir de l'amant face à l'indifférence de sa dame, le poussant à envisager la mort comme libération de ses souffrances. La première tâche de la composition d'un motet consiste à choisir le fragment du plein chant. Ici, le tenor « Obediens usque ad mortem » est extrait du graduel de la messe du Jeudi saint, inspiré de l'épître de saint Paul aux Philippiens (2, 8-9). Machaut façonne le tenor en deux colores (énoncé mélodique) qui coïncident chacun avec six taleae (énoncé rythmique), délimitant la pièce en deux parties. Le second color est en diminution, les valeurs rythmiques du tenor se retrouvent divisées par deux. La construction du tenor est la base de toute la structure de la pièce, ses caractéristiques modales et mélodiques déterminent l'œuvre entière ${ }^{4}$.

\begin{tabular}{|c|cccccc|cccccc|}
\hline Color & \multicolumn{5}{|c|}{ A } & \multicolumn{5}{c|}{ B } \\
\hline Talea & I & II & III & IV & V & VI & 1 & 2 & 3 & 4 & 5 & 6 \\
\hline
\end{tabular}

D’après Anna Maria Busse Berger ${ }^{5}$, la musique du haut Moyen Âge reste fortement marquée par une tradition orale, malgré le développement progressif de la notation ${ }^{6}$. Ainsi, de nombreux procédés sont utilisés afin de faciliter la mémorisation. Les modes rythmiques sont un moyen mnémotechnique tout comme la versification utilisée aux $\mathrm{XII}^{\mathrm{e}}$ et XIII ${ }^{\mathrm{e}}$ siècle. Pour Jean de Garlande ${ }^{7}$, les motifs rythmiques représentés par des figures précises indiquent le rythme modal. Chaque motif est donc à la base de la conception globale du temps dans la pièce. Il n'est pas étonnant de trouver, dans les voix supérieures du motet, trois cellules rythmiques récurrentes, qui peuvent évoquer le cœur de la conception modale du temps issue de l'oralité. On peut considérer que ces cellules rythmiques permettent d'appuyer la mémoire musicale ${ }^{8}$. 


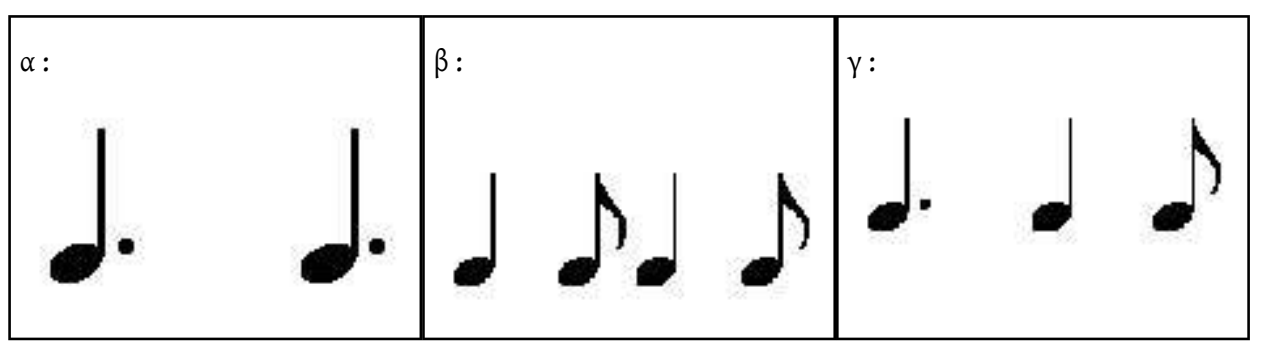

4 Dans le même but mnémotechnique, Machaut a construit sa talea de façon à ce que la dernière valeur qui la compose soit un silence. Ceci délimite très clairement la fin de la talea et met en évidence le passage de l'une à l'autre. Il se combine avec une tenue au motetus chevauchant l'enchainement de deux phrases rythmiques. L'association de ces deux éléments semble servir de point de repère lors de l'exécution par les chanteurs, chacun se synchronisant en fonction - voir par exemple le passage encadré entre le taleae III-IV, m. 36-37. De ce fait, la notion de mnémotechnique pourrait englober à la fois l'idée de mémorisation individuelle et de mise en place collective 9 .

5 Le motet est organisé en deux parties qui correspondent aux deux colores. Elles se distinguent par l'utilisation de procédés d'écriture différents. La diminution et le hoquet caractérisent la seconde partie. Ce jeu rythmique faisant alterner minimes et silences entre les voix supérieures est souvent employé dans les motets de l'Ars nova pour souligner la structure du tenor ${ }^{10}$. Dans le motet 10 , le hoquet ( $« \mathrm{~h}$.» en partition) met en avant chaque fin de talea. Sa présence est signalée par l'occurrence d'une note longue en homorythmie aux motetus et tenor dans les deux premières taleae et aux trois voix dans les taleae suivantes. L'agencement rythmique du hoquet est repris à l'identique à chaque apparition, ce qui constitue un marqueur évident de l'architecture musicale de la pièce. Ainsi, cet ensemble joue la même fonction que le couple «tenue/ silence » de la première partie.

6 Le texte du tenor, "Obediens usque ad mortem », est extrait de l'épître de saint Paul aux Philippiens ${ }^{11}:$ «Il s'est abaissé devenant obéissant jusqu'à la mort, et à la mort sur une croix, et c'est pourquoi Dieu l'a souverainement élevé et lui a conféré le nom qui est audessus de tout nom. "

7 C'est l'expression de la Kénose, mouvement d'abaissement par lequel Jésus renie sa condition divine pour rejoindre l'humanité et vivre dans l'obéissance de la foi jusqu'à la mort. La Résurrection, mouvement d'élévation, est la conséquence directe de la Kénose : le Christ, en acceptant cette condition inférieure, s'est vu ensuite élevé au rang ultime.

8 Les vers du triplum exploitent le champ lexical de l'amour : "ardant désir », " amours ", « espoir », «feu », puis du désespoir : «dolereus», « desespoir », «morir ». À six reprises, le feu est mentionné et renvoie à l'image de l'amant consumé par l'ardent désir puis par la douleur. Le motetus, quant à lui, décrit la douleur d'un amour sans espoir et le désir de mort : « Helas! », « mors », " desespoir », « l'ardour de mon triste desir », « sans espoir ».

9 La superposition des trois textes rend la compréhension difficile. Cependant, on observe une mise en valeur de certains mots par des silences ou des tenues aux autres voix (en gras dans les textes). Les éléments ainsi isolés agissent comme des mots-clés permettant à l'auditeur de saisir le propos du motet dans son ensemble ${ }^{12}$.

10 L'amant du motet est consumé par le feu de l'amour pour sa dame. Dans la religion chrétienne, le feu est associé à la purification de l'âme et le Christ est fréquemment 
dépeint comme "dame Sagesse » au Moyen Âge ${ }^{13}$. La recherche obstinée de la dame par l'amant est donc analogue à celle du Christ par le disciple chrétien. En envisageant la mort, évoquée dans le triplum et le motetus, l'amant accepte l'abaissement énoncé par la voix du tenor.

De façon didactique, Machaut nous entraîne d'une vision lyrique de l'amour courtois à une vision théologique. L'interaction des différents textes conduit à donner un sens spirituel aux vers profanes. La musique fait écho à cette interaction en entremêlant les voix musicales par différents procédés.

Premier procédé, l'omniprésence des cellules rythmiques récurrentes citées précédemment. Outre leur fonction mnémotechnique, elles confèrent une cohérence stylistique aux deux voix supérieures.

Conséquence de leur utilisation répétée, on note l'existence d'échanges rythmiques entre le motetus et le triplum : une cellule énoncée à l'une des voix est reprise par la suite à l'autre voix. Cette technique est poussée à l'extrême avec l'emploi d'un canon rythmique au début de la seconde partie - voir en partition, encadrés m. 73-76, B1.

Les procédés développés sur le plan rythmique le sont également sur le plan mélodique. En effet, on dénombre plusieurs échanges mélodiques entre les voix supérieures, procédé que l'on retrouve dans la messe de Notre-Dame ${ }^{14}$. De plus, l'omniprésence de cellules mélodiques en « $\mathrm{v}$ » ou « $\mathrm{v}$ inversé » peut être mise en parallèle avec la recherche d'une cohérence stylistique.

Le contour de ces deux cellules, aux profils opposés, rejoint la symbolique de la Kénose. La cellule en " $\mathrm{V}$ » suivant une ligne descendante puis ascendante, reproduit le mouvement de l'abaissement de l'âme suivi de l'élévation. A contrario, le "v inversé » renvoie au parcours de l'amant, transcendé par la joie de l'amour puis anéanti par la désillusion sentimentale. À deux reprises, ces cellules se retrouvent superposées, formant une figure en miroir. Chaque cellule représente respectivement la Kénose et l'amour charnel. Leur superposition nous conduit à nouveau vers l'idée d'un sens caché des textes profanes révélé par le texte liturgique - voir partition m. 62, encadré en pointillé.

Si l'on compare le déroulement littéraire du triplum et du motetus avec le déroulement musical du motet, on observe que les deux premiers tiers des textes se déploient sur le premier color. Le second color en diminution accompagne le dernier tiers des poèmes dont le sens est centré sur la mort. La mort joue en effet un rôle essentiel, constituant le noyau autour duquel tout s'articule. Elle est simultanément l'horizon de l'amant désespéré et la clé de la résurrection du Christ. Elle constitue un lien fort qui uni la lecture profane à la lecture liturgique.

17 Dans son ouvrage sur les motets de Machaut, Marie-Bernadette Dufourcet considère que le motet 10 est construit suivant trois taleae par color ${ }^{15}$. Or, dans le second color, le hoquet délimite très clairement six parties, qui correspondent à six taleae. L'ensemble de la pièce est donc structuré en six taleae par color.

Cependant, le palindrome envisagé par Dufourcet dans ce qu'elle considère comme une talea reste pertinent. En effet, son palindrome présente un silence comme axe de symétrie entre deux énoncés rythmiques qui sont en fait les taleae. Or, on observe un palindrome au sein même d'une talea. Il est donc évident de trouver un palindrome entre deux taleae séparées par un silence. 


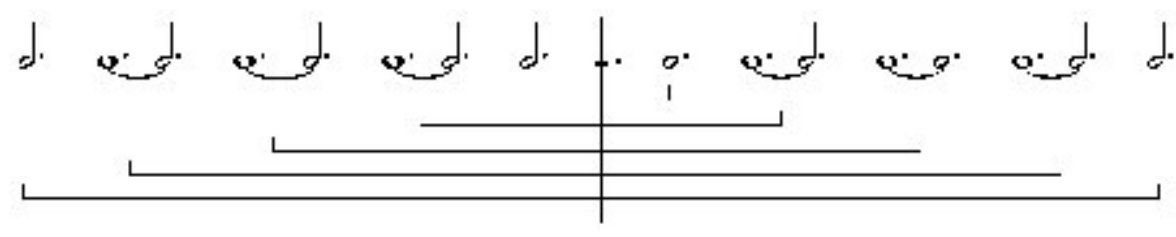

Palindrome vu par Dufurcet

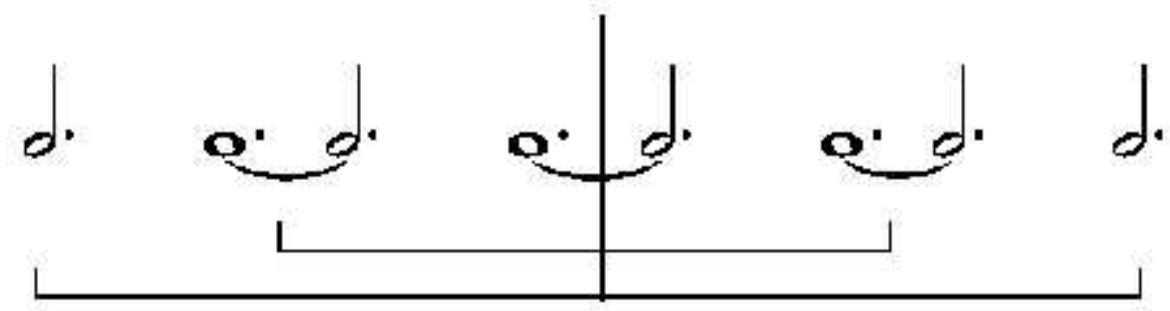

Palindrome au sein d'une talea

De plus, le chiffre six est porteur d'une symbolique primordiale. D'après Vincent Foster Hopper ${ }^{16}$, le six représente le nombre nuptial féminin tout en étant le premier nombre parfait: $1+2+3$. Ceci nous renvoie au double sens des textes, le nombre nuptial féminin fait référence à la dame désirée tandis que l'idée de perfection s'apparente au divin. Outre la construction du tenor en six taleae, le poème du triplum s'organise autour de quatre strophes de six vers délimitées par des couples de rimes refrain sur la sonorité -ure.

\section{AAAA $\underline{B B}$ - CCCC $\underline{B B}$ - DDDD $\underline{B B}$ - EEEE $\underline{B B}$}

Les trois premiers couples de rimes-refrain sont associés à une phrase rythmique qui revient à l'identique et accentue cet agencement par groupe de six vers - voir R dans la partition. Encore une fois, le texte et la musique sont étroitement liés. Il est intéressant d'observer que Machaut utilise le même procédé dans son motet 15 Amours qui a le pouoir / Faus Samblant m'a deceü / Vidi dominum en utilisant la même rime en -ure pour structurer le poème du triplum ${ }^{17}$. De plus, le motet 15 est lié au motet 10 par l'emploi de mots similaires et organisés suivant un plan structurel identique.

Motet 15, vers 23-24:

«Mais aveuc tous ces meschiés

Sueffre Amours qui est mes chiés »

Motet 10, vers 19-20:

«Et, pour croistre mes dolereus meschiés

Met dedens moy Amours, qui est mes chiés »

La construction du second color, dans lequel la thématique de la mort est centrale, est très nettement régie par le chiffre six. Les six taleae se déploient sur une durée de six brèves et accompagnent les six derniers vers du triplum et du motetus. Sachant que l'écart entre le nombre de vers des deux poèmes est important, il n'est pas anodin que pour cette partie, le même nombre de vers soit utilisé aux deux voix.

La présence du nombre six dans le second color en diminution découle directement de celle du nombre douze dans le premier color, conséquence du rapport de $1 / 2$ provoqué par la diminution. Le motet contient douze taleae au total et on recense douze brèves dans chaque taleae du premier color. De plus, le texte du motetus compte cent vingt syllabes et celui du triplum, deux cent quarante. Enfin, le premier et le treizième vers du triplum sont mis en relief par une phrase mélodique et rythmique singulière, réexposée 
à l'identique, qui délimite clairement à l'audition deux parties égales de douze vers. L'omniprésence du nombre douze mérite que l'on s'intéresse à sa symbolique.

Selon Hopper ${ }^{18}$, ce nombre évoque l'idée de cycle, tout comme la Kénose, où Jésus Christ, de condition divine, s'abaisse à vivre tel un homme avant de réintégrer son statut initial. Robertson démontre également que chaque motet s'inscrit dans un ensemble dont l'ordre aurait été voulu par Machaut et qui correspondrait au voyage spirituel chrétien ${ }^{19}$. Il serait intéressant d'envisager une analyse de chacun d'entre eux, pour émettre l'hypothèse d'un rapport entre le nombre douze et une logique de cycle musical.

En plus d'utiliser deux nombres symboliques, Machaut intègre le nombre d'or à l'architecture de sa pièce dans une optique de perfection. Sur le plan littéraire, on le trouve dans le rapport des vingt-quatre vers du triplum avec les quinze vers du motetus. Un mot joue le rôle de marqueur d'une section dorée. Mesure 41, on trouve le mot « que » superposé aux voix supérieures et souligné par une brève en homorythmie aux deux voix. Fait exceptionnel puisque c'est le seul moment dans le motet où un mot se trouve énoncé simultanément aux deux voix et traité rythmiquement à l'identique. La partie précédant ce mot s'étend sur une durée de quarante brèves, puis la pièce se poursuit sur un temps de soixante-cinq brèves. Du rapport de ces deux valeurs, on obtient le nombre d'or ${ }^{20}$.

Ayant pris connaissance des nombreux travaux musicologiques réfutant l'existence volontaire du nombre d'or dans les œuvres musicales de l'époque ${ }^{21}$, nous avons malgré tout choisi de considérer son existence dans ce motet. Au vu du caractère unique de la synchronisation du mot "que» aux deux voix supérieures et de son traitement homorythmique, le mettant incontestablement en valeur, il paraitrait improbable d'avoir à faire à une pure coïncidence. De plus, Machaut étant lui-même l'auteur de ses textes, il a consciemment décidé du nombre de vers les constituant, preuve d'un emploi volontaire du nombre d'or dans ce motet.

D'autre part, Jack Goody démontre que l'une des conséquences de l'écriture était l'invention de jeux de nombres, inimaginables dans une conception orale de l'œuvre ${ }^{22}$. Machaut conçoit ses pièces de manière figée, contrôlant avec précision chaque détail de l'œuvre. Ceci joue une fois de plus en faveur d'une utilisation intentionnelle du nombre d'or. Cependant, l'écriture précise du motet ne contredit en rien la présence de procédés mnémotechniques issus de la tradition musicale du temps. Enfin, la volonté de Machaut de diriger l'élaboration des manuscrits du cycle de ses motets, nous prouve à nouveau le désir du compositeur de garder une emprise forte sur ses créations. Il conserve ainsi intacte toute la puissance symbolique de sa poésie révélée et enrichie par la beauté de sa musique.

Intrigués par les nombreuses similitudes révélées entre les motets 10 et 15 , il nous apparaît à présent nécessaire d'étendre l'analyse à tous les motets de Machaut. Ceci permettrait d'enrichir l'hypothèse de Robertson d'un sens spirituel à l'ensemble, par une cohérence stylistique, symbolique et poétique. 
INDEX

Mots-clés : amour courtois, Guillaume de Machaut, kénose 